\title{
Global Solutions of 3-D Inhomogeneous Navier-Stokes System with Large Viscosity in One Variable
}

\section{Tiantian $\mathrm{Hao}^{*}$}

Academy of Mathematics \& Systems Science, Chinese Academy of Sciences, Beijing 100190, China, and School of Mathematical Sciences, University of Chinese Academy of Sciences, Beijing 100049, China.

Received 30 March 2021; Accepted 19 June 2021

\begin{abstract}
We consider the global well-posedness of three dimensional incompressible inhomogeneous Navier-Stokes equation with different viscous coefficients in the vertical and horizontal variables. In particular, when one of these viscous coefficients is large enough compared with the initial data and the initial density is close enough to a positive constant, we prove the global wellposedness of this system. This result extends the previous results in $[9,11]$ for the classical Navier-Stokes system.
\end{abstract}

AMS subject classifications: 35Q30, 76D03

Key words: Inhomogeneous Navier-Stokes system, anisotropic Littlewood-Paley theory, global well-posedness.

\section{Introduction}

We consider the global existence of strong solution to the following 3-D inhomogeneous incompressible anisotropic Navier-Stokes equations with initial density being sufficiently close to a positive constant in the critical space, $\dot{B}_{p, 1}^{\frac{3}{p}}\left(\mathbb{R}^{3}\right)$ for some $p \in] 3,4$, and with a large viscous coefficient in one direction:

${ }^{*}$ Corresponding author. Email address: htt@amss.ac.cn (T. Hao) 


$$
\left\{\begin{array}{l}
\partial_{t} \rho+u \cdot \nabla \rho=0, \quad(t, x) \in \mathbb{R}^{+} \times \mathbb{R}^{3}, \\
\rho\left(\partial_{t} u+u \cdot \nabla u\right)-v_{\mathrm{h}} \Delta_{\mathrm{h}} u-v_{\mathrm{v}} \partial_{3}^{2} u+\nabla P=0, \\
\operatorname{div} u=0, \\
\left.(\rho, u)\right|_{t=0}=\left(\rho_{0}, u_{0}\right),
\end{array}\right.
$$

where $\rho, u$ stand for the density and velocity of the fluid respectively, $\nabla P$ is a scalar pressure function, which guarantees the divergence free condition of the velocity field, $v_{\mathrm{h}}$ and $v_{\mathrm{v}}$ are viscous coefficients so that $v_{\mathrm{v}}$ is much larger than $v_{\mathrm{h}}>0, \Delta_{\mathrm{h}} \stackrel{\text { def }}{=} \partial_{x_{1}}^{2}+\partial_{x_{2}}^{2}$ designates the horizontal Laplacian. One may check [8] for more background of this system. The system (1.1) has three major basic features. First, the incompressibility expressed by fact that the vector field $u$ is divergence free gives

$$
\|\rho(t)\|_{L^{\infty}}=\left\|\rho_{0}\right\|_{L^{\infty}} .
$$

Second, this system has the following enenrgy law

$$
\frac{1}{2}\|\sqrt{\rho} u(t)\|_{L^{2}}^{2}+v_{\mathrm{h}}\left\|\nabla_{\mathrm{h}} u\right\|_{L_{t}^{2}\left(L^{2}\right)}^{2}+v_{\mathrm{v}}\left\|\partial_{3} u\right\|_{L_{t}^{2}\left(L^{2}\right)}^{2}=\frac{1}{2}\left\|\sqrt{\rho_{0}} u_{0}\right\|_{L^{2}}^{2} .
$$

The third basic feature is the scaling invariance property: If $(\rho, u, P)$ is a solution of $(1.1)$ on $[0, T] \times \mathbb{R}^{3}$, then the rescaled triplet $(\rho, u, P)_{\lambda}$ defined by

$$
(\rho, u, P)_{\lambda}(t, x) \stackrel{\text { def }}{=}\left(\rho\left(\lambda^{2} t, \lambda x\right), \lambda u\left(\lambda^{2} t, \lambda x\right), \lambda^{2} P\left(\lambda^{2} t, \lambda x\right)\right) \quad \text { with } \quad \lambda \in \mathbb{R}^{+}
$$

is also a solution of (1.1) on $\left[0, T / \lambda^{2}\right] \times \mathbb{R}^{3}$. Motivated by (1.3), Danchin [7] established the well-posedness of (1.1) in the so-called critical functional framework for small perturbations of some positive constant density.

When the density $\rho$ is away from zero, we set $a \stackrel{\text { def }}{=} \frac{1}{\rho}-1$. Then (1.1) can be equivalently formulated as

$$
\left\{\begin{array}{l}
\partial_{t} a+u \cdot \nabla a=0, \quad(t, x) \in \mathbb{R}^{+} \times \mathbb{R}^{3} \\
\partial_{t} u+u \cdot \nabla u-v_{\mathrm{h}}(1+a) \Delta_{\mathrm{h}} u-v_{\mathrm{v}}(1+a) \partial_{3}^{2} u+(1+a) \nabla P=0 \\
\operatorname{div} u=0 \\
\left.(a, u)\right|_{t=0}=\left(a_{0}, u_{0}\right)
\end{array}\right.
$$

One may check [5] and the references therein concerning the well-posedness theory of the system (1.1) or (1.4). In particular, when $\rho_{0} \in L^{\infty}$ with a positive lower bound and initial velocity being sufficiently small in the critical Besove space, 
$B_{2,1}^{\frac{1}{2}}$, Zhang [13] proved the global existence of weak solutions to the system (1.1) and also provided a lower bound for the lifespan of smooth enough solutions of (1.1).

Motivated by $[9,11]$ concerning the global wellposedness of 3-D anisotropic incompressible Navier-Stokes system with different viscous coefficients in the vertical and horizontal variables, we are going to study the inhomogeneous Navier-Stokes system, which also has different viscous coefficients. Our result below (see Theorem 1.1) ensures that given initial data $u_{0}$, for small enough $a_{0}$, if $v_{\mathrm{v}}$ is sufficiently large compared with $v_{\mathrm{h}}>0$, then (1.4) has a unique global solution.

Notation. In what follows, we shall denote

$$
B_{p}^{s} \stackrel{\text { def }}{=} B_{p, 1}^{s}, \quad B_{p}^{s_{1}, s_{2}} \stackrel{\text { def }}{=} B_{p, 1}^{s_{1}, s_{2}} .
$$

The definitions of the above spaces will be recalled in Section 2.

The main result states as follows:

Theorem 1.1. Let $p \in] 3,4[$ and $\theta \in] 3 / 4,1\left[\right.$. Let $a_{0} \in B_{p}^{\frac{3}{p}}\left(\mathbb{R}^{3}\right)$ and $u_{0} \in B_{2}^{0, \frac{1}{2}}\left(\mathbb{R}^{3}\right) \cap H^{1}\left(\mathbb{R}^{3}\right)$ be a solenoidal vector field. Then there exist two positive constants $c_{0}$ and $C$ such that if

$$
\begin{aligned}
& \left(v_{\mathrm{h}}\left\|a_{0}\right\|_{B_{p}^{\frac{3}{p}}}+\left\|a_{0}\right\|_{B_{p}^{\frac{3}{p}}}\left\|u_{0}\right\|_{B_{2}^{0, \frac{1}{2}}}+\frac{v_{\mathrm{h}}^{1-\theta}}{v_{\mathrm{v}}^{1-\theta}}\left\|a_{0}\right\|_{B_{p}^{\frac{3}{p}}}\left\|u_{0}\right\|_{B_{2}^{\frac{1}{2}}}\right. \\
& \left.\quad+\frac{1}{v_{\mathrm{h}}^{\theta} v_{\mathrm{v}}^{1-\theta}}\left\|u_{0}\right\|_{B_{2}^{0, \frac{1}{2}}}\left(\left\|u_{0}\right\|_{B_{2}^{0, \frac{1}{2}}}+\left\|u_{0}\right\|_{B_{2}^{\frac{1}{2}}}\right)\right) \exp \left(\frac{C\left(\left\|u_{0}\right\|_{B_{2}^{0, \frac{1}{2}}}+\left\|u_{0}\right\|_{B_{2}^{\frac{1}{2}}}\right)}{v_{\mathrm{h}}^{\theta} v_{\mathrm{v}}^{1-\theta}}\right) \leq c_{0} v_{\mathrm{h}},
\end{aligned}
$$

the system (1.4) has a unique global solution

$$
a \in \mathcal{C}\left([0, \infty) ; B_{p}^{\frac{3}{p}}\left(\mathbb{R}^{3}\right)\right), \quad u \in \mathcal{C}\left([0, \infty) ; H^{1}\left(\mathbb{R}^{3}\right)\right) \cap L^{2}\left(\mathbb{R}^{+} ; \dot{H}^{2}\left(\mathbb{R}^{3}\right)\right) .
$$

This paper is organized as follows. In Section 2, we shall recall some basic facts on Littlewood-Paley theory and some direct consequences. In Section 3, we shall prove a regularity criteria, namely (3.2), for the local smooth solution constructed in [12]. In Section 4, we shall prove that under the assumption of (1.5), the regularity criteria (3.2) holds for any $T<T^{*}$, where $T^{*}$ denotes the lifespan of the local smooth solution of (1.1). In order to do so, we denote $u_{L} \stackrel{\text { def }}{=} e^{t\left(v_{\mathrm{h}} \Delta_{\mathrm{h}}+v_{\mathrm{v}} \partial_{3}^{2}\right)} u_{0}$. Then $u_{L}$ solves

$$
\left\{\begin{array}{l}
\partial_{t} u_{L}-v_{\mathrm{h}} \Delta_{\mathrm{h}} u_{L}-v_{\mathrm{v}} \partial_{3}^{2} u_{L}=0, \quad(t, x) \in \mathbb{R}^{+} \times \mathbb{R}^{3}, \\
\left.u_{L}\right|_{t=0}=u_{0} .
\end{array}\right.
$$


Motivated by [11], we write

$$
u=u_{L}+R,
$$

then it follows from (1.4) and (1.6), that

$$
\left\{\begin{array}{l}
\partial_{t} R+R \cdot \nabla R-v_{\mathrm{h}} \Delta_{\mathrm{h}} R-v_{\mathrm{v}} \partial_{3}^{2} R+\nabla P=F \quad \text { with } \\
F=a\left(v_{\mathrm{h}} \Delta_{\mathrm{h}} R+v_{\mathrm{v}} \partial_{3}^{2} R+v_{\mathrm{h}} \Delta_{\mathrm{h}} u_{L}+v_{\mathrm{v}} \partial_{3}^{2} u_{L}\right) \\
\quad-\quad u_{L} \cdot \nabla u_{L}-R \cdot \nabla u_{L}-u_{L} \cdot \nabla R-a \nabla P \\
\operatorname{div} R=0 \\
\left.R\right|_{t=0}=0
\end{array}\right.
$$

Indeed we shall prove that the regularity criteria (3.2) can not happen for the remaining term $R$ through anisotropic Littlewood-Paley analysis.

\section{Preliminaries}

Before we present the function spaces we are going to work with in this context, let us briefly recall some basic facts on Littlewood-Paley theory (see, e.g., [1]). Let $\varphi$ and $\chi$ be smooth functions supported in $\mathcal{C} \stackrel{\text { def }}{=}\left\{\tau \in \mathbb{R}^{+}, \frac{3}{4} \leq \tau \leq \frac{8}{3}\right\}$ and $\mathfrak{B} \stackrel{\text { def }}{=}\left\{\tau \in \mathbb{R}^{+}, \tau \leq \frac{4}{3}\right\}$ respectively such that

$$
\begin{array}{ll}
\sum_{j \in \mathbb{Z}} \varphi\left(2^{-j} \tau\right)=1 & \text { for } \quad \tau>0 \\
\chi(\tau)+\sum_{j \geq 0} \varphi\left(2^{-j} \tau\right)=1 & \text { for } \quad \tau \geq 0
\end{array}
$$

For $a \in \mathcal{S}^{\prime}\left(\mathbb{R}^{3}\right)$, we set

$$
\begin{aligned}
& \Delta_{k}^{\mathrm{h}} a \stackrel{\text { def }}{=} \mathcal{F}^{-1}\left(\varphi\left(2^{-k}\left|\xi_{\mathrm{h}}\right|\right) \widehat{a}\right), \quad S_{k}^{\mathrm{h}} a \stackrel{\text { def }}{=} \mathcal{F}^{-1}\left(\chi\left(2^{-k}\left|\xi_{\mathrm{h}}\right|\right) \widehat{a}\right), \\
& \Delta_{\ell}^{\mathrm{v}} a \stackrel{\text { def }}{=} \mathcal{F}^{-1}\left(\varphi\left(2^{-\ell}\left|\xi_{3}\right|\right) \widehat{a}\right), \quad S_{\ell}^{\mathrm{v}} a \stackrel{\text { def }}{=} \mathcal{F}^{-1}\left(\chi\left(2^{-\ell}\left|\xi_{3}\right|\right) \widehat{a}\right), \\
& \Delta_{j} a \stackrel{\text { def }}{=} \mathcal{F}^{-1}\left(\varphi\left(2^{-j}|\xi|\right) \widehat{a}\right), \quad S_{j} a \stackrel{\text { def }}{=} \mathcal{F}^{-1}\left(\chi\left(2^{-j}|\xi|\right) \widehat{a}\right),
\end{aligned}
$$

where $\xi_{\mathrm{h}}=\left(\xi_{1}, \xi_{2}\right), \xi_{=}=\left(\xi_{\mathrm{h}}, \xi_{3}\right), \mathcal{F} a$ and $\widehat{a}$ denote the Fourier transform of the distribution $a$. The dyadic operators satisfy the property of almost orthogonality

$$
\begin{array}{lll}
\Delta_{k} \Delta_{j} a \equiv 0, & \text { if } & |k-j| \geq 2, \\
\Delta_{k}\left(S_{j-1} a \Delta_{j} b\right) \equiv 0, & \text { if } & |k-j| \geq 5 .
\end{array}
$$


Similar properties hold for $\Delta_{k}^{\mathrm{h}}$ and $\Delta_{\ell}^{\mathrm{v}}$.

Let us recall the anisotropic Bernstein lemma from $[3,10]$.

Lemma 2.1. Let $\mathcal{B}_{\mathrm{h}}\left(\right.$ resp. $\mathcal{B}_{\mathrm{V}}$ ) be a ball of $\mathbb{R}_{\mathrm{h}}^{2}$ (resp. $\mathbb{R}_{\mathrm{v}}$ ), and $\mathcal{C}_{\mathrm{h}}$ (resp. $\mathcal{C}_{\mathrm{V}}$ ) be a ring of $\mathbb{R}_{\mathrm{h}}^{2}$ (resp. $\mathbb{R}_{\mathrm{v}}$ ); let $1 \leq p_{2} \leq p_{1} \leq \infty$ and $1 \leq q_{2} \leq q_{1} \leq \infty$. Then there hold:

- If Supp $\widehat{a} \subset 2^{k} \mathcal{B}_{\mathrm{h}}$, then $\left\|\partial_{x_{\mathrm{h}}}^{\alpha} a\right\|_{L_{\mathrm{h}}^{p_{1}}\left(L_{\mathrm{v}}^{q_{1}}\right)} \lesssim 2^{k\left(|\alpha|+2\left(1 / p_{2}-1 / p_{1}\right)\right)}\|a\|_{L_{\mathrm{h}}^{p_{2}}\left(L_{\mathrm{v}}^{q_{1}}\right)}$.

- If Supp $\widehat{a} \subset 2^{\ell} \mathcal{B}_{\mathrm{V}}$, then $\left\|\partial_{x_{3}}^{\beta} a\right\|_{L_{\mathrm{h}}^{p_{1}}\left(L_{\mathrm{v}}^{q_{1}}\right)} \lesssim 2^{\ell\left(\beta+\left(1 / q_{2}-1 / q_{1}\right)\right)}\|a\|_{L_{\mathrm{h}}^{p_{1}}\left(L_{\mathrm{V}}^{q_{2}}\right)}$.

- If Supp $\widehat{a} \subset 2^{k} \mathcal{C}_{\mathrm{h}}$, then $\|a\|_{L_{\mathrm{h}}^{p_{1}}\left(L_{\mathrm{v}}^{q_{1}}\right)} \lesssim 2^{-k N} \sup _{|\alpha|=N}\left\|\partial_{x_{\mathrm{h}}}^{\alpha} a\right\|_{L_{\mathrm{h}}^{p_{1}}\left(L_{\mathrm{v}}^{q_{1}}\right)}$.

- If Supp $\widehat{a} \subset 2^{\ell} \mathcal{C}_{\mathrm{v}}$, then $\|a\|_{L_{\mathrm{h}}^{p_{1}}\left(L_{\mathrm{v}}^{q_{1}}\right)} \lesssim 2^{-\ell N}\left\|\partial_{x_{3}}^{N} a\right\|_{L_{\mathrm{h}}^{p_{1}}\left(L_{\mathrm{v}}^{q_{1}}\right)}$.

Due to the anisotropic spectral properties of the linear equation (1.6), we need the following anisotropic Besov norm:

Definition 2.1. Let $s, s_{1}, s_{2} \in \mathbb{R}, 1 \leq p \leq \infty, 1 \leq q \leq \infty$ and $a \in \mathcal{S}_{h}^{\prime}\left(\mathbb{R}^{3}\right)$, we define the norm

$$
\begin{aligned}
& \|a\|_{B_{p, 1}^{s}} \stackrel{\text { def }}{=}\left(2^{j s}\left\|\Delta_{j} a\right\|_{L^{p}}\right)_{\ell^{1}}{ }^{\prime} \\
& \|a\|_{B_{p, 1}^{s_{1}, s_{2}}} \stackrel{\text { def }}{=}\left(2^{\ell s_{2}} 2^{k s_{1}}\left\|\Delta_{k}^{\mathrm{h}} \Delta_{\ell^{\mathrm{v}}}^{\mathrm{v}} a\right\|_{L^{p}}\right)_{\ell^{1^{\prime}}}, \\
& \|a\|_{\tilde{L}_{t}^{q}\left(B_{p, 1}^{s}\right)} \stackrel{\text { def }}{=}\left(2^{j s}\left\|\Delta_{j} a\right\|_{L^{q}\left([0, t] ; L^{p}\right)}\right)_{\ell^{1^{\prime}}}, \\
& \|a\|_{\tilde{L}_{t}^{q}\left(B_{p, 1}^{s_{1}, s_{2}}\right)} \stackrel{\text { def }}{=}\left(2^{\ell s_{2}} 2^{k s_{1}}\left\|\Delta_{k}^{\mathrm{h}} \Delta_{\ell}^{\mathrm{v}} a\right\|_{L^{q}\left([0, t] ; L^{p}\right)}\right)_{\ell^{1}} .
\end{aligned}
$$

We recall the classical homogeneous anisotropic Sobolev norm as follows:

$$
\|a\|_{\dot{H}^{s_{1}, s_{2}}} \stackrel{\text { def }}{=}\left(\sum_{(k, \ell) \in \mathbb{Z}^{2}} 2^{2 k s_{1}} 2^{2 \ell s_{2}}\left\|\Delta_{k}^{\mathrm{h}} \Delta_{\ell}^{\mathrm{v}} a\right\|_{L^{2}}^{2}\right)^{\frac{1}{2}} .
$$

For the convenience of readers, we also recall the following product laws in the anisotropic spaces.

Lemma 2.2 ([2]). Let $s_{1} \leq \frac{2}{p}, s_{2} \leq \frac{2}{p}$, with $s_{1}+s_{2}>0$. Let $\sigma_{1} \leq \frac{1}{p}, \sigma_{2} \leq \frac{1}{p}$, with $\sigma_{1}+\sigma_{2}>0$. If $a \in B_{p}^{s_{1}, \sigma_{1}}\left(\mathbb{R}^{3}\right), b \in B_{p}^{s_{2}, \sigma_{2}}\left(\mathbb{R}^{3}\right)$, then $a b \in B_{p}^{s_{1}+s_{2}-\frac{2}{p}, \sigma_{1}+\sigma_{2}-\frac{1}{p}}\left(\mathbb{R}^{3}\right)$, and

$$
\|a b\|_{B_{p}^{s_{1}+s_{2}-\frac{2}{p}, \sigma_{1}+\sigma_{2}-\frac{1}{p}}} \lesssim\|a\|_{B_{p}^{s_{1}, \sigma_{1}}}\|b\|_{B_{p}^{s_{2}, \sigma_{2}}}
$$


As an application of the above facts, we present the following lemma concerning the solution of the linear equation (1.6), which tells us the small quantities that will be used in what follows.

Lemma 2.3. Let $p \in] 3,4[, \theta \in] 3 / 4,1\left[\right.$, and $u_{0} \in B_{2}^{0, \frac{1}{2}} \cap B_{2}^{\frac{1}{2}}$. Let $u_{L} \stackrel{\text { def }}{=} e^{t\left(v_{\mathrm{h}} \Delta_{\mathrm{h}}+v_{\mathrm{v}} \partial_{3}^{2}\right)} u_{0}$. Then we have

$$
\begin{aligned}
\left\|u_{L}\right\|_{\tilde{L}_{t}^{\infty}\left(B_{p}^{\left.-1+\frac{2}{p}, \frac{1}{p}\right)}\right.} \lesssim\left\|u_{0}\right\|_{B_{2}^{0, \frac{1}{2}}{ }^{\prime}} \\
\left\|\nabla_{\mathrm{h}} u_{L}\right\|_{L_{t}^{1}\left(B_{p}^{\left.\frac{2}{p}, \frac{1}{p}\right)}\right.} \lesssim \frac{1}{v_{\mathrm{h}}^{\theta} v_{\mathrm{V}}^{1-\theta}}\left\|u_{0}\right\|_{B_{2}^{\frac{1}{2}}} \\
\left\|\partial_{3} u_{L}\right\|_{L_{t}^{1}\left(B_{p}^{\frac{2}{p}, \frac{1}{p}}\right)} \lesssim \frac{1}{\sqrt{v_{\mathrm{h}} v_{\mathrm{v}}}}\left\|u_{0}\right\|_{B_{2}^{0, \frac{1}{2}}} \\
\left\|\partial_{3}^{2} u_{L}\right\|_{L_{t}^{1}\left(B_{p}^{\left.-1+\frac{2}{p}, \frac{1}{p}\right)}\right.} \lesssim \frac{1}{v_{\mathrm{v}}}\left\|u_{0}\right\|_{B_{2}^{0, \frac{1}{2}}} \cdot
\end{aligned}
$$

Proof. By the definition of $u_{L}$, we have

$$
\left\|\Delta_{k}^{\mathrm{h}} \Delta_{\ell}^{\mathrm{v}} u_{L}\right\|_{L_{t}^{\infty}\left(L^{p}\right)} \lesssim\left\|\Delta_{k}^{\mathrm{h}} \Delta_{\ell}^{\mathrm{v}} u_{0}\right\|_{L^{p}} \lesssim 2^{k\left(1-\frac{2}{p}\right)} 2^{\ell\left(\frac{1}{2}-\frac{1}{p}\right)}\left\|\Delta_{k}^{\mathrm{h}} \Delta_{\ell}^{\mathrm{v}} u_{0}\right\|_{L^{2}}
$$

which implies (2.4a).

We also observe that

$$
\begin{aligned}
\left\|\Delta_{k}^{\mathrm{h}} \Delta_{\ell}^{\mathrm{v}} u_{L}\right\|_{L_{t}^{1}\left(L^{p}\right)} & \lesssim\left\|\Delta_{k}^{\mathrm{h}} \Delta_{\ell}^{\mathrm{v}} u_{0}\right\|_{L^{p}} \int_{0}^{t} e^{-c t^{\prime}\left(v_{\mathrm{h}} 2^{2 k}+v_{\mathrm{v}} 2^{2 \ell}\right)} d t^{\prime} \\
& \lesssim \frac{1}{v_{\mathrm{h}} 2^{2 k}+v_{\mathrm{v}} 2^{2 \ell}}\left\|\Delta_{k}^{\mathrm{h}} \Delta_{\ell}^{\mathrm{v}} u_{0}\right\|_{L^{p}}
\end{aligned}
$$

which together with Lemma 2.1 ensures that

$$
\left\|\Delta_{k}^{\mathrm{h}} \Delta_{\ell}^{\mathrm{v}} u_{L}\right\|_{L_{t}^{1}\left(L^{p}\right)} \lesssim \frac{2^{k\left(1-\frac{2}{p}\right)} 2^{\ell\left(\frac{1}{2}-\frac{1}{p}\right)}}{v_{\mathrm{h}} 2^{2 k}+v_{\mathrm{v}} 2^{2 \ell}}\left\|\Delta_{k}^{\mathrm{h}} \Delta_{\ell}^{\mathrm{v}} u_{0}\right\|_{L^{2}}
$$

Thanks to (2.5), for any $\theta \in[0,1]$, we infer

$$
\begin{aligned}
\left\|\nabla_{\mathrm{h}} \Delta_{k}^{\mathrm{h}} \Delta_{\ell}^{\mathrm{v}} u_{L}\right\|_{L_{t}^{1}\left(L^{p}\right)} & \lesssim \frac{2^{k\left(2-\frac{2}{p}\right)} 2^{\ell\left(\frac{1}{2}-\frac{1}{p}\right)}}{v_{\mathrm{h}}^{\theta} \nu_{\mathrm{v}}^{1-\theta} 2^{2 k \theta} 2^{2 \ell(1-\theta)}}\left\|\Delta_{k}^{\mathrm{h}} \Delta_{\ell}^{\mathrm{v}} u_{0}\right\|_{L^{2}} \\
& \lesssim \frac{2^{k\left(2-\frac{2}{p}-2 \theta\right)} 2^{\ell\left(2 \theta-\frac{3}{2}-\frac{1}{p}\right)}}{v_{\mathrm{h}}^{\theta} v_{\mathrm{v}}^{1-\theta}}\left\|\Delta_{k}^{\mathrm{h}} \Delta_{\ell}^{\mathrm{v}} u_{0}\right\|_{L^{2}}
\end{aligned}
$$


which together with $[4$, Lemma 4.3] implies that for $\theta \in] 3 / 4,1[$

$$
\left\|\nabla_{\mathrm{h}} u_{L}\right\|_{L_{t}^{1}\left(B_{p}^{\frac{2}{p}, \frac{1}{p}}\right)} \lesssim \frac{1}{v_{\mathrm{h}}^{\theta} v_{\mathrm{v}}^{1-\theta}}\left\|u_{0}\right\|_{B^{2-2 \theta, 2 \theta-\frac{3}{2}}} \lesssim \frac{1}{v_{\mathrm{h}}^{\theta} v_{\mathrm{v}}^{1-\theta}}\left\|u_{0}\right\|_{B_{2}^{\frac{1}{2}}} .
$$

This proves $(2.4 b)$.

Along the same line, we have

$$
\begin{gathered}
\left\|\partial_{3} \Delta_{k}^{\mathrm{h}} \Delta_{\ell}^{\mathrm{v}} u_{L}\right\|_{L_{t}^{1}\left(L^{p}\right)} \lesssim \frac{2^{k\left(1-\frac{2}{p}\right)} 2^{\ell\left(\frac{3}{2}-\frac{1}{p}\right)}}{v_{\mathrm{h}} 2^{2 k}+v_{\mathrm{v}} 2^{2 \ell}}\left\|\Delta_{k}^{\mathrm{h}} \Delta_{\ell}^{\mathrm{v}} u_{0}\right\|_{L^{2}} \\
\lesssim \frac{2^{k\left(1-\frac{2}{p}\right)} 2^{\ell\left(\frac{3}{2}-\frac{1}{p}\right)}}{\sqrt{v_{\mathrm{h}} \nu_{\mathrm{v}}} 2^{k} 2^{\ell}}\left\|\Delta_{k}^{\mathrm{h}} \Delta_{\ell}^{\mathrm{v}} u_{0}\right\|_{L^{2}} \lesssim \frac{2^{-\frac{2}{p} k} 2^{\ell\left(\frac{1}{2}-\frac{1}{p}\right)}}{\sqrt{v_{\mathrm{h}} \nu_{\mathrm{v}}}}\left\|\Delta_{k}^{\mathrm{h}} \Delta_{\ell}^{\mathrm{v}} u_{0}\right\|_{L^{2}}
\end{gathered}
$$

which leads to $(2.4 \mathrm{c})$.

Similarly, we observe that

$$
\begin{aligned}
\left\|\partial_{3}^{2} \Delta_{k}^{\mathrm{h}} \Delta_{\ell}^{\mathrm{v}} u_{L}\right\|_{L_{t}^{1}\left(L^{p}\right)} & \lesssim \frac{2^{k\left(1-\frac{2}{p}\right)} 2^{\ell\left(\frac{5}{2}-\frac{1}{p}\right)}}{v_{\mathrm{h}} 2^{2 k}+v_{\mathrm{v}} 2^{2 \ell}}\left\|\Delta_{k}^{\mathrm{h}} \Delta_{\ell}^{\mathrm{v}} u_{0}\right\|_{L^{2}} \\
& \lesssim \frac{2^{k\left(1-\frac{2}{p}\right)} 2^{\ell\left(\frac{1}{2}-\frac{1}{p}\right)}}{v_{\mathrm{v}}}\left\|\Delta_{k}^{\mathrm{h}} \Delta_{\ell}^{\mathrm{v}} u_{0}\right\|_{L^{2}}
\end{aligned}
$$

which ensures (2.4d). This completes the proof of Lemma 2.3.

Remark 2.1. We deduce from the law of product Lemmas 2.2 and 2.3 that for any $\theta \in] 3 / 4,1[$

$$
\begin{aligned}
& \left\|u_{L} \cdot \nabla u_{L}\right\|_{L_{t}^{1}\left(B_{p}^{-1+\frac{2}{p}, \frac{1}{p}}\right)} \\
& \lesssim\left\|u_{L}\right\|_{\tilde{L}_{t}^{\infty}\left(B_{p}^{\left.-1+\frac{2}{p}, \frac{1}{p}\right)}\right.}\left(\left\|\nabla_{\mathrm{h}} u_{L}\right\|_{L_{t}^{1}\left(B_{p}^{\frac{2}{p}, \frac{1}{p}}\right)}+\left\|\partial_{3} u_{L}\right\|_{L_{t}^{1}\left(B_{p}^{\frac{2}{p}, \frac{1}{p}}\right)}\right) \\
& \lesssim \frac{\left\|u_{0}\right\|_{B_{2}^{0, \frac{1}{2}}}^{2}}{\sqrt{v_{\mathrm{h}} v_{\mathrm{v}}}}+\frac{\left\|u_{0}\right\|_{B_{2}^{0, \frac{1}{2}}}\left\|u_{0}\right\|_{B_{2}^{\frac{1}{2}}}}{v_{\mathrm{h}}^{\theta} v_{\mathrm{v}}^{1-\theta}} \lesssim \frac{\left\|u_{0}\right\|_{B_{2}^{0, \frac{1}{2}}}\left(\left\|u_{0}\right\|_{B_{2}^{0, \frac{1}{2}}}+\left\|u_{0}\right\|_{B_{2}^{\frac{1}{2}}}\right)}{v_{\mathrm{h}}^{\theta} v_{\mathrm{v}}^{1-\theta}} .
\end{aligned}
$$

Finally, we also recall a lemma concerning the propagation of regularities for the transport equation.

Lemma $2.4([1])$. Let $p \in] 3,4\left[\right.$. Then given $a_{0}$ in $B_{p}^{\frac{3}{p}}\left(\mathbb{R}^{3}\right)$ and a vector field $u$ in $L^{1}([0, T]$; $\operatorname{Lip}\left(\mathbb{R}^{3}\right)$ ) with $\operatorname{div} u=0$, the following transport equation

$$
\partial_{t} a+u \cdot \nabla a=0,\left.\quad a\right|_{t=0}=a_{0}
$$


has a unique solution a satisfying

$$
\|a\|_{\tilde{L}_{t}^{\infty}\left(B_{p}^{\frac{3}{p}}\right)} \leq\left\|a_{0}\right\|_{B_{p}^{\frac{3}{p}}} \exp \left(C \int_{0}^{t}\left\|\nabla u\left(t^{\prime}\right)\right\|_{L^{\infty} d t^{\prime}}\right) .
$$

\section{The regularity criteria}

In this section, we investigate the regularity criteria for the system (1.1).

Proposition 3.1. Let $\left(\rho_{0}, u_{0}\right)$ satisfy $0<m \leq \rho_{0} \leq M$ and $u_{0} \in H^{1}\left(\mathbb{R}^{3}\right)$. Let $(\rho, u)$ be the unique solution of the system (1.1) on $[0, T[$ which satisfies

$$
m \leq \rho \leq M \quad \text { and } \quad u \in C\left(\left[0, T\left[; H^{1}\left(\mathbb{R}^{3}\right)\right) \cap L^{2}(] 0, T\left[; \dot{H}^{2}\left(\mathbb{R}^{3}\right)\right) .\right.\right.
$$

If

$$
\int_{0}^{T}\left(\|u(t)\|_{L^{\infty}}^{2}+\|\nabla u(t)\|_{L^{\infty}}\right) d t<+\infty
$$

then $(\rho, u)$ may be continued beyond $T$.

Proof. We remark that the unique solution $(\rho, u)$ of the system (1.1) which satisfies (3.1) has been constructed in [12]. In what follows, we just present the a priori estimates. Without loss of generality, we may assume that $v_{\mathrm{v}}>v_{\mathrm{h}}$. As a convention in the rest of this section, we shall always denote by $C$ a constant depending on $m$ and $M$ in (3.1), which may be different from line to line. We first get, by taking $L^{2}$ inner product of momentum equation of (1.1) with $u$, that

$$
\frac{1}{2} \frac{d}{d t}\|\sqrt{\rho} u(t)\|_{L^{2}}^{2}+v_{\mathrm{h}}\left\|\nabla_{h} u\right\|_{L^{2}}^{2}+v_{\mathrm{v}}\left\|\partial_{3} u\right\|_{L^{2}}^{2}=0
$$

Integrating the above equality over $[0, t]$ yields (1.2).

We split the rest of the proof into the following two steps.

Step 1. $\dot{H}^{1}$ energy estimate of $u$.

By taking $L^{2}$ inner product of (1.1) with $\partial_{t} u$, we obtain

$$
\left\|\sqrt{\rho} u_{t}\right\|_{L^{2}}^{2}+\frac{1}{2} \frac{d}{d t}\left(v_{\mathrm{h}}\left\|\nabla_{\mathrm{h}} u\right\|_{L^{2}}^{2}+v_{\mathrm{v}}\left\|\partial_{3} u\right\|_{L^{2}}^{2}\right) \leq\left|\left(\rho u \cdot \nabla u \mid u_{t}\right)\right| .
$$

Observing that

$$
\begin{aligned}
\left|\left(\rho u \cdot \nabla u \mid u_{t}\right)\right| & \leq C\left\|\sqrt{\rho} u_{t}\right\|_{L^{2}}\|u\|_{L^{\infty}}\|\nabla u\|_{L^{2}} \\
& \leq \frac{1}{2}\left\|\sqrt{\rho} u_{t}\right\|_{L^{2}}^{2}+C\|u\|_{L^{\infty}}^{2}\|\nabla u\|_{L^{2}}^{2} \\
& \leq \frac{1}{2}\left\|\sqrt{\rho} u_{t}\right\|_{L^{2}}^{2}+\frac{C}{v_{\mathrm{h}}}\|u\|_{L^{\infty}}^{2}\left(v_{\mathrm{h}}\left\|\nabla_{\mathrm{h}} u\right\|_{L^{2}}^{2}+v_{\mathrm{v}}\left\|\partial_{3} u\right\|_{L_{2}}^{2}\right) .
\end{aligned}
$$


By inserting the above estimate into (3.4) and then applying Gronwall inequality to the resulting inequality, we find

$$
\begin{aligned}
& v_{\mathrm{h}}\left\|\nabla_{\mathrm{h}} u\right\|_{L_{t}^{\infty}\left(L^{2}\right)}^{2}+v_{\mathrm{v}}\left\|\partial_{3} u\right\|_{L_{t}^{\infty}\left(L^{2}\right)}^{2}+\left\|\sqrt{\rho} u_{t}\right\|_{L_{t}^{2}\left(L^{2}\right)}^{2} \\
\leq & \left(v_{\mathrm{h}}\left\|\nabla_{\mathrm{h}} u_{0}\right\|_{L^{2}}^{2}+v_{\mathrm{v}}\left\|\partial_{3} u_{0}\right\|_{L^{2}}^{2}\right) \exp \left(\frac{C}{v_{\mathrm{h}}} \int_{0}^{t}\|u\|_{L^{\infty}}^{2} d t^{\prime}\right) .
\end{aligned}
$$

On the other hand, in view of (1.1), we write

$$
-v_{\mathrm{h}} \Delta_{\mathrm{h}} u-v_{\mathrm{v}} \partial_{3}^{2} u+\nabla P=-\rho\left(u_{t}+u \cdot \nabla u\right),
$$

from which and the classical estimates on the Stokes system, we infer

$$
\begin{aligned}
& v_{\mathrm{h}}\left\|\nabla_{\mathrm{h}}^{2} u\right\|_{L^{2}}+v_{\mathrm{v}}\left\|\partial_{3}^{2} u\right\|_{L^{2}}+\|\nabla P\|_{L^{2}} \\
\leq C & \left(\left\|\rho u_{t}\right\|_{L^{2}}+\|\rho u \cdot \nabla u\|_{L^{2}}\right) \leq C\left(\left\|\rho u_{t}\right\|_{L^{2}}+\|u\|_{L^{\infty}}\|\nabla u\|_{L^{2}}\right)
\end{aligned}
$$

which together with (3.5) ensures that

$$
\begin{aligned}
& v_{\mathrm{h}}\left\|\nabla_{\mathrm{h}}^{2} u\right\|_{L_{t}^{2}\left(L^{2}\right)}^{2}+v_{\mathrm{v}}\left\|\partial_{3}^{2} u\right\|_{L_{t}^{2}\left(L^{2}\right)}^{2}+\|\nabla P\|_{L_{t}^{2}\left(L^{2}\right)}^{2} \\
\leq & \frac{1}{v_{\mathrm{h}}}\left(v_{\mathrm{h}}\left\|\nabla_{\mathrm{h}} u_{0}\right\|_{L^{2}}^{2}+v_{\mathrm{v}}\left\|\partial_{3} u_{0}\right\|_{L^{2}}^{2}\right) \exp \left(\frac{C}{v_{\mathrm{h}}} \int_{0}^{t}\|u\|_{L^{\infty}}^{2} d t^{\prime}\right) .
\end{aligned}
$$

Step 2. $\dot{H}^{2}$ energy estimate of $u$.

We first get, by taking the time derivative to the momentum equation (1.1), that

$$
\rho\left(u_{t t}+u \cdot \nabla u_{t}\right)-v_{\mathrm{h}} \Delta_{\mathrm{h}} u_{t}-v_{\mathrm{v}} \partial_{3}^{2} u_{t}+\nabla P_{t}=-\rho_{t}\left(u_{t}+u \cdot \nabla u\right)-\rho u_{t} \cdot \nabla u .
$$

Taking the $L^{2}$ inner product of the above equation with $u_{t}$, we write

$$
\begin{aligned}
& \frac{1}{2} \frac{d}{d t}\left\|\sqrt{\rho} u_{t}\right\|_{L^{2}}^{2}+v_{\mathrm{h}}\left\|\nabla_{\mathrm{h}} u_{t}\right\|_{L^{2}}^{2}+v_{\mathrm{v}}\left\|\partial_{3} u_{t}\right\|_{L^{2}}^{2} \\
&=-\left(\rho u_{t} \cdot \nabla u \mid u_{t}\right)-\left(\rho_{t} u_{t} \mid u_{t}\right)-\left(\rho_{t} u \cdot \nabla u \mid u_{t}\right) \\
& \stackrel{\text { def }}{=} E+F+G .
\end{aligned}
$$

Obviously, we have

$$
|E| \lesssim\|\nabla u\|_{L^{\infty}}\left\|\sqrt{\rho} u_{t}\right\|_{L^{2}}^{2}
$$


While noticing that $\rho_{t}=-u \cdot \nabla \rho$, by using integration by parts, we get

$$
\begin{aligned}
F & =-2 \int_{\mathbb{R}^{3}}\left(\rho u \cdot \nabla u_{t} \mid u_{t}\right) d x \leq C\|u\|_{L^{\infty}}\left\|\nabla u_{t}\right\|_{L^{2}}\left\|\sqrt{\rho} u_{t}\right\|_{L^{2}} \\
& \leq \frac{v_{\mathrm{h}}}{4}\left\|\nabla u_{t}\right\|_{L^{2}}^{2}+\frac{C}{v_{\mathrm{h}}}\|u\|_{L^{\infty}}^{2}\left\|\sqrt{\rho} u_{t}\right\|_{L^{2}}^{2} .
\end{aligned}
$$

To deal with $G$, by using integrating by parts, we have

$$
\begin{aligned}
G= & \int_{\mathbb{R}^{3}}\left((\rho u \cdot \nabla u) \cdot \nabla u \mid u_{t}\right) d x+\int_{\mathbb{R}^{3}}\left((\rho u \otimes u): \nabla^{2} u \mid u_{t}\right) d x \\
& +\int_{\mathbb{R}^{3}}\left((\rho u \cdot \nabla u) \mid u \cdot \nabla u_{t}\right) d x \\
& \stackrel{\text { def }}{=} G_{1}+G_{2}+G_{3} .
\end{aligned}
$$

Applying Sobolev inequality gives

$$
G_{1} \leq C\|u\|_{L^{6}}\|\nabla u\|_{L^{6}}^{2}\left\|\sqrt{\rho} u_{t}\right\|_{L^{2}} \leq C\|\nabla u\|_{L^{2}}\left\|\nabla^{2} u\right\|_{L^{2}}^{2}\left\|\sqrt{\rho} u_{t}\right\|_{L^{2}},
$$

which together with (3.6) ensures that

$$
\begin{aligned}
G_{1} & \leq \frac{C}{v_{\mathrm{h}}}\|\nabla u\|_{L^{2}}\left\|\nabla^{2} u\right\|_{L^{2}}\left(\left\|\rho u_{t}\right\|_{L^{2}}+\|u\|_{L^{\infty}}\|\nabla u\|_{L^{2}}\right)\left\|\sqrt{\rho} u_{t}\right\|_{L^{2}} \\
& \leq \frac{C}{v_{\mathrm{h}}}\left(\|u\|_{L^{\infty}}^{2}+\|\nabla u\|_{L^{2}}^{2}+\left\|\nabla^{2} u\right\|_{L^{2}}^{2}\right)\left\|\sqrt{\rho} u_{t}\right\|_{L^{2}}^{2}+\frac{C}{v_{\mathrm{h}}}\|\nabla u\|_{L^{2}}^{4}\left\|\nabla^{2} u\right\|_{L^{2}}^{2} .
\end{aligned}
$$

While along the same line to the proof of (3.6), one has

$$
\begin{aligned}
& v_{\mathrm{h}}\left\|\nabla_{\mathrm{h}}^{2} u\right\|_{L^{2}}+v_{\mathrm{v}}\left\|\partial_{3}^{2} u\right\|_{L^{2}}+\|\nabla P\|_{L^{2}} \\
\leq & C\left(\left\|\sqrt{\rho} u_{t}\right\|_{L^{2}}+\|u \cdot \nabla u\|_{L^{2}}\right) \leq \frac{C}{v_{\mathrm{h}}}\left(\left\|\sqrt{\rho} u_{t}\right\|_{L^{2}}+\|\nabla u\|_{L^{2}}^{3}\right)+\frac{v_{\mathrm{h}}}{2}\left\|\nabla^{2} u\right\|_{L^{2}}
\end{aligned}
$$

which implies

$$
v_{\mathrm{h}}\left\|\nabla_{\mathrm{h}}^{2} u\right\|_{L^{2}}+v_{\mathrm{v}}\left\|\partial_{3}^{2} u\right\|_{L^{2}}+\|\nabla P\|_{L^{2}} \leq \frac{C}{v_{\mathrm{h}}}\left(\left\|\sqrt{\rho} u_{t}\right\|_{L^{2}}+\|\nabla u\|_{L^{2}}^{3}\right) .
$$

Hence we obtain

$$
\begin{aligned}
G_{2} & \leq C\|u\|_{L^{\infty}}^{2}\left\|\nabla^{2} u\right\|_{L^{2}}\left\|\sqrt{\rho} u_{t}\right\|_{L^{2}} \\
& \leq \frac{C}{v_{\mathrm{h}}^{2}}\|u\|_{L^{\infty}}^{2}\left(\left\|\sqrt{\rho} u_{t}\right\|_{L^{2}}+\|\nabla u\|_{L^{2}}^{3}\right)\left\|\sqrt{\rho} u_{t}\right\|_{L^{2}} \\
& \leq \frac{C}{v_{\mathrm{h}}^{2}}\|u\|_{L^{\infty}}^{2}\left(\left\|\sqrt{\rho} u_{t}\right\|_{L^{2}}^{2}+\|\nabla u\|_{L^{2}}^{6}\right) .
\end{aligned}
$$


Finally we observe that

$$
\begin{aligned}
G_{3} & \leq C\|u\|_{L^{6}}^{2}\|\nabla u\|_{L^{6}}\left\|\nabla u_{t}\right\|_{L^{2}} \leq C\|\nabla u\|_{L^{2}}^{2}\left\|\nabla^{2} u\right\|_{L^{2}}\left\|\nabla u_{t}\right\|_{L^{2}} \\
& \leq \frac{v_{\mathrm{h}}}{4}\left\|\nabla u_{t}\right\|_{L^{2}}^{2}+\frac{C}{v_{\mathrm{h}}}\|\nabla u\|_{L^{2}}^{4}\left\|\nabla^{2} u\right\|_{L^{2}}^{2} .
\end{aligned}
$$

By inserting the above estimates into (3.8), we conclude that

$$
\begin{aligned}
& \frac{d}{d t}\left\|\sqrt{\rho} u_{t}\right\|_{L^{2}}^{2}+v_{\mathrm{h}}\left\|\nabla_{\mathrm{h}} u_{t}\right\|_{L^{2}}^{2}+v_{\mathrm{v}}\left\|\partial_{3} u_{t}\right\|_{L^{2}}^{2} \\
\lesssim & \left(\|\nabla u\|_{L^{\infty}}+\frac{1}{v_{\mathrm{h}}^{2}}\|u\|_{L^{\infty}}^{2}+\frac{1}{v_{\mathrm{h}}}\|\nabla u\|_{L^{2}}^{2}+\frac{1}{v_{\mathrm{h}}}\left\|\nabla^{2} u\right\|_{L^{2}}^{2}\right)\left\|\sqrt{\rho} u_{t}\right\|_{L^{2}}^{2} \\
& +\frac{1}{v_{\mathrm{h}}}\|\nabla u\|_{L^{2}}^{4}\left\|\nabla^{2} u\right\|_{L^{2}}^{2}+\frac{1}{v_{\mathrm{h}}^{2}}\|u\|_{L^{\infty}}^{2}\|\nabla u\|_{L^{2}}^{6} .
\end{aligned}
$$

Due to the initial data $u_{0} \in H^{1}$ instead of $H^{2}$, as in [12], we introduce $\sigma(t) \stackrel{\text { def }}{=}$ $\min (1, t)$ multiply the above inequality by $\sigma(t)$ to get

$$
\begin{aligned}
& \quad \frac{d}{d t}\left(\sigma(t)\left\|\sqrt{\rho} u_{t}\right\|_{L^{2}}^{2}\right)+\sigma(t)\left(v_{\mathrm{h}}\left\|\nabla_{\mathrm{h}} u_{t}\right\|_{L^{2}}^{2}+v_{\mathrm{v}}\left\|\partial_{3} u_{t}\right\|_{L^{2}}^{2}\right) \\
& \lesssim\left\|\sqrt{\rho} u_{t}\right\|_{L^{2}}^{2}+\sigma(t)\left(\|\nabla u\|_{L^{\infty}}+\frac{1}{v_{\mathrm{h}}^{2}}\|u\|_{L^{\infty}}^{2}+\frac{1}{v_{\mathrm{h}}}\|\nabla u\|_{L^{2}}^{2}+\frac{1}{v_{\mathrm{h}}}\left\|\nabla^{2} u\right\|_{L^{2}}^{2}\right)\left\|\sqrt{\rho} u_{t}\right\|_{L^{2}}^{2} \\
& \quad+\frac{\sigma(t)}{v_{\mathrm{h}}}\|\nabla u\|_{L^{2}}^{4}\left\|\nabla^{2} u\right\|_{L^{2}}^{2}+\frac{\sigma(t)}{v_{\mathrm{h}}^{2}}\|u\|_{L^{\infty}}^{2}\|\nabla u\|_{L^{2}}^{6} .
\end{aligned}
$$

Applying Gronwall inequality to above estimate and using the fact $\sigma(t) \leq 1$, we obtain

$$
\begin{gathered}
\sigma(t)\left\|\sqrt{\rho} u_{t}\right\|_{L^{2}}^{2}+\int_{0}^{t} \sigma\left(t^{\prime}\right)\left(v_{\mathrm{h}}\left\|\nabla_{\mathrm{h}} u_{t}\right\|_{L^{2}}^{2}+v_{\mathrm{v}}\left\|\partial_{3} u_{t}\right\|_{L^{2}}^{2}\right) d t^{\prime} \\
\lesssim\left(\left\|\sqrt{\rho} u_{t}\right\|_{L_{t}^{2}\left(L^{2}\right)}^{2}+\int_{0}^{t}\left(\frac{1}{v_{\mathrm{h}}}\|\nabla u\|_{L^{2}}^{4}\left\|\nabla^{2} u\right\|_{L^{2}}^{2}+\frac{1}{v_{\mathrm{h}}^{2}}\|u\|_{L^{\infty}}^{2}\|\nabla u\|_{L^{2}}^{6}\right) d t^{\prime}\right) \\
\quad \times \exp \int_{0}^{t}\left(\|\nabla u\|_{L^{\infty}}+\frac{1}{v_{\mathrm{h}}^{2}}\|u\|_{L^{\infty}}^{2}+\frac{1}{v_{\mathrm{h}}}\left\|\nabla^{2} u\right\|_{L^{2}}^{2}+\frac{1}{v_{\mathrm{h}}}\|\nabla u\|_{L^{2}}^{2}\right) d t^{\prime} .
\end{gathered}
$$

By using (1.2), (3.5) and (3.7), we can get

$$
\int_{0}^{t} \frac{1}{v_{\mathrm{h}}}\|\nabla u\|_{L^{2}}^{4}\left\|\nabla^{2} u\right\|_{L^{2}}^{2} d t^{\prime} \leq \frac{1}{v_{\mathrm{h}}}\|\nabla u\|_{L_{t}^{\infty}\left(L^{2}\right)}^{4}\left\|\nabla^{2} u\right\|_{L_{t}^{2}\left(L^{2}\right)}^{2}
$$




$$
\begin{aligned}
\leq & \frac{1}{v_{\mathrm{h}}^{5}}\left(v_{\mathrm{h}}^{3}\left\|\nabla_{\mathrm{h}} u_{0}\right\|_{L^{2}}^{6}+v_{\mathrm{v}}^{3}\left\|\partial_{3} u_{0}\right\|_{L^{2}}^{6}\right) \exp \left(\frac{C}{v_{\mathrm{h}}} \int_{0}^{t}\|u\|_{L^{\infty}}^{2} d t^{\prime}\right), \\
& \int_{0}^{t} \frac{1}{v_{\mathrm{h}}^{2}}\|u\|_{L^{\infty}}^{2}\|\nabla u\|_{L^{2}}^{6} d t^{\prime} \leq \frac{1}{v_{\mathrm{h}}^{2}}\|\nabla u\|_{L_{t}^{\infty}\left(L^{2}\right)}^{6} \int_{0}^{t}\|u\|_{L^{\infty}}^{2} d t^{\prime} \\
\leq & \frac{1}{v_{\mathrm{h}}^{5}}\left(v_{\mathrm{h}}^{3}\left\|\nabla_{\mathrm{h}} u_{0}\right\|_{L^{2}}^{6}+v_{\mathrm{v}}^{3}\left\|\partial_{3} u_{0}\right\|_{L^{2}}^{6}\right) \exp \left(\frac{C}{v_{\mathrm{h}}} \int_{0}^{t}\|u\|_{L^{\infty}}^{2} d t^{\prime}\right) .
\end{aligned}
$$

By substituting (3.5) and the above estimates into (3.11), we achieve

$$
\begin{aligned}
& \left\|\sigma(t) \sqrt{\rho} u_{t}\right\|_{L_{t}^{\infty}\left(L^{2}\right)}^{2}+\int_{0}^{t} \sigma\left(t^{\prime}\right)\left(v_{\mathrm{h}}\left\|\nabla_{\mathrm{h}} u_{t}\right\|_{L^{2}}^{2}+v_{\mathrm{v}}\left\|\partial_{3} u_{t}\right\|_{L^{2}}^{2}\right) d t^{\prime} \\
\leq & \frac{1}{v_{\mathrm{h}}^{5}}\left(v_{\mathrm{h}}^{3}\left\|\nabla_{\mathrm{h}} u_{0}\right\|_{L^{2}}^{6}+v_{\mathrm{v}}^{3}\left\|\partial_{3} u_{0}\right\|_{L^{2}}^{6}\right) \exp \left(\frac{C}{v_{\mathrm{h}}^{2}} \int_{0}^{t}\left(\|u\|_{L^{\infty}}^{2}+\|\nabla u\|_{L^{\infty}}\right) d t^{\prime}\right) \\
& \times \exp \left(\frac{1}{v_{\mathrm{h}}^{2}}\left\|\sqrt{\rho_{0}} u_{0}\right\|_{L^{2}}^{2}+\frac{1}{v_{\mathrm{h}}^{3}}\left(v_{\mathrm{h}}\left\|\nabla_{\mathrm{h}} u_{0}\right\|_{L^{2}}^{2}+v_{\mathrm{v}}\left\|\partial_{3} u_{0}\right\|_{L^{2}}^{2}\right) \exp \left(\int_{0}^{t} \frac{1}{v_{\mathrm{h}}}\|u\|_{L^{\infty}}^{2} d t^{\prime}\right)\right),
\end{aligned}
$$

which together with (3.9) ensures that

$$
\begin{aligned}
& v_{\mathrm{h}}\left\|\sigma(t) \nabla_{\mathrm{h}}^{2} u\right\|_{L_{t}^{\infty}\left(L^{2}\right)}^{2}+v_{\mathrm{v}}\left\|\sigma(t) \partial_{3}^{2} u\right\|_{L_{t}^{\infty}\left(L^{2}\right)}^{2}+\|\sigma(t) \nabla P\|_{L_{t}^{\infty}\left(L^{2}\right)}^{2} \\
\leq & \frac{1}{v_{\mathrm{h}}^{8}}\left(v_{\mathrm{h}}^{3}\left\|\nabla_{\mathrm{h}} u_{0}\right\|_{L^{2}}^{6}+v_{\mathrm{v}}^{3}\left\|\partial_{3} u_{0}\right\|_{L^{2}}^{6}\right) \exp \left(\frac{C}{v_{\mathrm{h}}^{2}} \int_{0}^{t}\left(\|u\|_{L^{\infty}}^{2}+\|\nabla u\|_{L^{\infty}}\right) d t^{\prime}\right) \\
& \quad \times \exp \left(\frac{1}{v_{\mathrm{h}}^{2}}\left\|\sqrt{\rho_{0}} u_{0}\right\|_{L^{2}}^{2}+\frac{1}{v_{\mathrm{h}}^{3}}\left(v_{\mathrm{h}}\left\|\nabla_{\mathrm{h}} u_{0}\right\|_{L^{2}}^{2}+v_{\mathrm{v}}\left\|\partial_{3} u_{0}\right\|_{L^{2}}^{2}\right) \exp \left(\int_{0}^{t} \frac{1}{v_{\mathrm{h}}}\|u\|_{L^{\infty}}^{2} d t^{\prime}\right)\right) .
\end{aligned}
$$

With the estimates (1.2), (3.5), (3.7) and (3.12), we can follow the same line to the proof of [12, Theorem 1.2] that under the assumption (3.2), we can prove the solution $(\rho, u)$ can be extended beyond T. Furthermore, by using Lagrangian approach, such a solution is unique. This completes the proof of Proposition 3.1.

\section{The proof of the Theorem 1.1}

The goal of this section is to present the proof of Theorem 1.1. Indeed according to Proposition 3.1, it remains to show that there holds (3.2) for any $T<T^{*}$, with $T^{*}$ being the lifespan of the solution $(a, u)$. In order to do this, we first present the estimate of the pressure function appearing in (1.7). 
Lemma 4.1. Let $p \in] 3,4[$. Then the pressure function $\nabla P$ determined by (1.7) satisfies

$$
\begin{aligned}
& \|\nabla P\|_{B_{p}^{-1+\frac{2}{p}, \frac{1}{p}}} \lesssim\|R \cdot \nabla R\|_{B_{p}^{-1+\frac{2}{p}, \frac{1}{p}}}+v_{\mathrm{h}}\|a\|_{B_{p}^{\frac{2}{p}, \frac{1}{p}}}\left(\left\|\Delta_{\mathrm{h}} R\right\|_{B_{p}^{-1+\frac{2}{p}, \frac{1}{p}}}+\left\|\Delta_{\mathrm{h}} u_{L}\right\|_{B_{p}^{-1+\frac{2}{p}, \frac{1}{p}}}\right) \\
& +v_{\mathrm{v}}\|a\|_{B_{p}^{\frac{2}{p}, \frac{1}{p}}}\left(\left\|\partial_{3}^{2} R\right\|_{B_{p}^{-1+\frac{2}{p}, \frac{1}{p}}}+\left\|\partial_{3}^{2} u_{L}\right\|_{B_{p}^{-1+\frac{2}{p}, \frac{1}{p}}}\right) \\
& +\|a\|_{B_{p}^{\frac{2}{p}, \frac{1}{p}}}\|\nabla P\|_{B_{p}^{-1+\frac{2}{p}, \frac{1}{p}}}+\left\|u_{L} \cdot \nabla u_{L}\right\|_{B_{p}^{-1+\frac{2}{p}, \frac{1}{p}}} \\
& +\left\|R \cdot \nabla u_{L}\right\|_{B_{p}^{-1+\frac{2}{p}, \frac{1}{p}}}+\left\|u_{L} \cdot \nabla R\right\|_{B_{p}^{-1+\frac{2}{p}, \frac{1}{p}}} .
\end{aligned}
$$

Proof. Applying the operator div to the $R$ equation of (1.7) yields

$$
\begin{aligned}
\Delta P=\operatorname{div}( & -R \cdot \nabla R+v_{\mathrm{h}} a \Delta_{\mathrm{h}} R+v_{\mathrm{v}} a \partial_{3}^{2} R+v_{\mathrm{h}} a \Delta_{\mathrm{h}} u_{L} \\
& \left.+v_{\mathrm{v}} a \partial_{3}^{2} u_{L}-a \nabla P-u_{L} \cdot \nabla u_{L}-R \cdot \nabla u_{L}-u_{L} \cdot \nabla R\right),
\end{aligned}
$$

we thus write

$$
\begin{aligned}
\nabla P=\nabla(-\Delta)^{-1} \operatorname{div}\left(R \cdot \nabla R-v_{\mathrm{h}} a \Delta_{\mathrm{h}} R-v_{\mathrm{v}} a \partial_{3}^{2} R-v_{\mathrm{h}} a \Delta_{\mathrm{h}} u_{L}\right. \\
\left.-v_{\mathrm{v}} a \partial_{3}^{2} u_{L}+a \nabla P+u_{L} \cdot \nabla u_{L}+R \cdot \nabla u_{L}+u_{L} \cdot \nabla R\right) .
\end{aligned}
$$

As $\nabla(-\Delta)^{-1} \operatorname{div}$ is a homogeneous Fourier multiplier of degree 0 , then by using the product laws, Lemma 2.2, we conclude the proof of (4.1).

Let us now present the proof of Theorem 1.1.

Proof of Theorem 1.1. Let $p \in] 3,4\left[\right.$. By applying $\Delta_{k}^{\mathrm{h}} \Delta_{\ell}^{\mathrm{v}}$ to the (1.7) and then taking $L^{2}$ inner product of the resulting momentum equation with $\left|\Delta_{k}^{\mathrm{h}} \Delta_{\ell}^{\mathrm{v}} R^{i}\right|^{p-2} \Delta_{k}^{\mathrm{h}} \Delta_{\ell}^{\mathrm{v}} R^{i}$, we get

$$
\begin{aligned}
& \quad \frac{1}{p} \frac{d}{d t}\left\|\Delta_{k}^{\mathrm{h}} \Delta_{\ell}^{\mathrm{v}} R^{i}\right\|_{L^{p}}^{p}-\int_{\mathbb{R}^{3}} \Delta_{k}^{\mathrm{h}} \Delta_{\ell}^{\mathrm{v}}\left(v_{\mathrm{h}} \Delta_{\mathrm{h}} R^{i}+v_{\mathrm{v}} \partial_{3}^{2} R^{i}\right)\left|\Delta_{k}^{\mathrm{h}} \Delta_{\ell}^{\mathrm{v}} R^{i}\right|^{p-2} \Delta_{k}^{\mathrm{h}} \Delta_{\ell}^{\mathrm{v}} R^{i} d x \\
& =-\int_{\mathbb{R}^{3}} \Delta_{k}^{\mathrm{h}} \Delta_{\ell}^{\mathrm{v}}\left(R \cdot \nabla R^{i}\right)\left|\Delta_{k}^{\mathrm{h}} \Delta_{\ell}^{\mathrm{v}} R^{i}\right|^{p-2} \Delta_{k}^{\mathrm{h}} \Delta_{\ell}^{\mathrm{v}} R^{i} d x \\
& \quad+v_{\mathrm{h}} \int_{\mathbb{R}^{3}} \Delta_{k}^{\mathrm{h}} \Delta_{\ell}^{\mathrm{v}}\left(a \Delta_{\mathrm{h}} R^{i}+a \Delta_{\mathrm{h}} u_{L}^{i}\right)\left|\Delta_{k}^{\mathrm{h}} \Delta_{\ell}^{\mathrm{v}} R^{i}\right|^{p-2} \Delta_{k}^{\mathrm{h}} \Delta_{\ell}^{\mathrm{v}} R^{i} d x \\
& \quad+v_{\mathrm{v}} \int_{\mathbb{R}^{3}} \Delta_{k}^{\mathrm{h}} \Delta_{\ell}^{\mathrm{v}}\left(a \partial_{3}^{2} R^{i}+a \partial_{3}^{2} u_{L}^{i}\right)\left|\Delta_{k}^{\mathrm{h}} \Delta_{\ell}^{\mathrm{v}} R^{i}\right|^{p-2} \Delta_{k}^{\mathrm{h}} \Delta_{\ell}^{\mathrm{v}} R^{i} d x \\
& \quad-\int_{\mathbb{R}^{3}} \Delta_{k}^{\mathrm{h}} \Delta_{\ell}^{\mathrm{v}}\left(u_{L} \cdot \nabla u_{L}^{i}+R \cdot \nabla u_{L}^{i}+u_{L} \cdot \nabla R^{i}\right)\left|\Delta_{k}^{\mathrm{h}} \Delta_{\ell}^{\mathrm{v}} R^{i}\right|^{p-2} \Delta_{k}^{\mathrm{h}} \Delta_{\ell}^{\mathrm{v}} R^{i} d x \\
& \quad-\int_{\mathbb{R}^{3}} \Delta_{k}^{\mathrm{h}} \Delta_{\ell}^{\mathrm{v}}\left((a+1) \partial_{i} P\right)\left|\Delta_{k}^{\mathrm{h}} \Delta_{\ell}^{\mathrm{v}} R^{i}\right|^{p-2} \Delta_{k}^{\mathrm{h}} \Delta_{\ell}^{\mathrm{v}} R^{i} d x .
\end{aligned}
$$


It follows from [6, Lemma A.5] that there exists a positive $\bar{c}$ so that

$$
\begin{aligned}
& -\int_{\mathbb{R}^{3}} \Delta_{\mathrm{h}} \Delta_{k}^{\mathrm{h}} \Delta_{\ell}^{\mathrm{v}} R^{(i)}\left|\Delta_{k}^{\mathrm{h}} \Delta_{\ell}^{\mathrm{v}} R^{(i)}\right|^{p-2} \Delta_{k}^{\mathrm{h}} \Delta_{\ell}^{\mathrm{v}} R^{(i)} d x \geq \bar{c} 2^{2 k}\left\|\Delta_{k}^{\mathrm{h}} \Delta_{\ell}^{\mathrm{v}} R^{(i)}\right\|_{L^{p}}^{p}, \\
& -\int_{\mathbb{R}^{3}} \partial_{3}^{2} \Delta_{k}^{\mathrm{h}} \Delta_{\ell}^{\mathrm{v}} R^{(i)}\left|\Delta_{k}^{\mathrm{h}} \Delta_{\ell}^{\mathrm{v}} R(i)\right|^{p-2} \Delta_{k}^{\mathrm{h}} \Delta_{\ell}^{\mathrm{v}} R^{(i)} d x \geq \bar{c} 2^{2 \ell}\left\|\Delta_{k}^{\mathrm{h}} \Delta_{\ell}^{\mathrm{v}} R^{(i)}\right\|_{L^{p}}^{p} .
\end{aligned}
$$

By inserting the above inequalities into (4.2), we get, by a standard derivation, that

$$
\begin{aligned}
& \left\|\Delta_{k}^{\mathrm{h}} \Delta_{\ell}^{\mathrm{v}} R\right\|_{L_{t}^{\infty}\left(L^{p}\right)}+v_{\mathrm{h}} 2^{2 k}\left\|\Delta_{k}^{\mathrm{h}} \Delta_{\ell}^{\mathrm{v}} R\right\|_{L_{t}^{1}\left(L^{p}\right)}+v_{\mathrm{v}} 2^{2 \ell}\left\|\Delta_{k}^{\mathrm{h}} \Delta_{\ell}^{\mathrm{v}} R\right\|_{L_{t}^{1}\left(L^{p}\right)} \\
& \lesssim\left\|\Delta_{k}^{\mathrm{h}} \Delta_{\ell}^{\mathrm{v}}(R \cdot \nabla R)\right\|_{L_{t}^{1}\left(L^{p}\right)}+v_{\mathrm{h}}\left\|\Delta_{k}^{\mathrm{h}} \Delta_{\ell}^{\mathrm{v}}\left(a \Delta_{\mathrm{h}} R\right)\right\|_{L_{t}^{1}\left(L^{p}\right)}+v_{\mathrm{v}}\left\|\Delta_{k}^{\mathrm{h}} \Delta_{\ell}^{\mathrm{v}}\left(a \partial_{3}^{2} R\right)\right\|_{L_{t}^{1}\left(L^{p}\right)} \\
& \quad+v_{\mathrm{h}}\left\|\Delta_{k}^{\mathrm{h}} \Delta_{\ell}^{\mathrm{v}}\left(a \Delta_{\mathrm{h}} u_{L}\right)\right\|_{L_{t}^{1}\left(L^{p}\right)}+v_{\mathrm{v}}\left\|\Delta_{k}^{\mathrm{h}} \Delta_{\ell}^{\mathrm{v}}\left(a \partial_{3}^{2} u_{L}\right)\right\|_{L_{t}^{1}\left(L^{p}\right)}+\left\|\Delta_{k}^{\mathrm{h}} \Delta_{\ell}^{\mathrm{v}}\left(u_{L} \cdot \nabla u_{L}\right)\right\|_{L_{t}^{1}\left(L^{p}\right)} \\
& \quad+\left\|\Delta_{k}^{\mathrm{h}} \Delta_{\ell}^{\mathrm{v}}\left(R \cdot \nabla u_{L}\right)\right\|_{L_{t}^{1}\left(L^{p}\right)}+\left\|\Delta_{k}^{\mathrm{h}} \Delta_{\ell}^{\mathrm{v}}\left(u_{L} \cdot \nabla R\right)\right\|_{L_{t}^{1}\left(L^{p}\right)}+\left\|\Delta_{k}^{\mathrm{h}} \Delta_{\ell}^{\mathrm{v}}((a+1) \nabla P)\right\|_{L_{t}^{1}\left(L^{p}\right)^{p}} \cdot
\end{aligned}
$$

By multiplying the above inequality by $2^{k\left(-1+\frac{2}{p}\right)} 2^{\frac{\ell}{p}}$ and then summing up the resulting inequalities for $k, \ell \in \mathbb{Z}$, we obtain

$$
\begin{aligned}
& \|R\|_{\tilde{L}_{t}^{\infty}\left(B_{p}^{-1+\frac{2}{p}, \frac{1}{p}}\right)}+v_{\mathrm{h}}\|R\|_{L_{t}^{1}\left(B_{p}^{1+\frac{2}{p}, \frac{1}{p}}\right)}+v_{\mathrm{v}}\|R\|_{L_{t}^{1}\left(B_{p}^{-1+\frac{2}{p}, 2+\frac{1}{p}}\right)} \\
& \lesssim\|R \cdot \nabla R\|_{L_{t}^{1}\left(B_{p}^{-1+\frac{2}{p}, \frac{1}{p}}\right)}+v_{\mathrm{h}}\|a\|_{\tilde{L}_{t}^{\infty}\left(B_{p}^{\frac{2}{p}, \frac{1}{p}}\right)}\left\|\Delta_{\mathrm{h}} R\right\|_{L_{t}^{1}\left(B_{p}^{-1+\frac{2}{p}, \frac{1}{p}}\right)} \\
& \quad+v_{\mathrm{v}}\|a\|_{\tilde{L}_{t}^{\infty}\left(B_{p}^{\frac{p}{p}, \frac{1}{p}}\right)}\left\|\partial_{3}^{2} R\right\|_{L_{t}^{1}\left(B_{p}^{-1+\frac{2}{p}, \frac{1}{p}}\right)}+v_{\mathrm{h}}\|a\|_{\tilde{L}_{t}^{\infty}\left(B_{p}^{\frac{2}{p}, \frac{1}{p}}\right)}\left\|\Delta_{\mathrm{h}} u_{L}\right\|_{L_{t}^{1}\left(B_{p}^{-1+\frac{2}{p}, \frac{1}{p}}\right)} \\
& \quad+v_{\mathrm{v}}\|a\|_{\tilde{L}_{t}^{\infty}\left(B_{p}^{\frac{2}{p}, \frac{1}{p}}\right)}\left\|\partial_{3}^{2} u_{L}\right\|_{L_{t}^{1}\left(B_{p}^{-1+\frac{2}{p}, \frac{1}{p}}\right)}+\left\|u_{L} \cdot \nabla u_{L}\right\|_{L_{t}^{1}\left(B_{p}^{-1+\frac{2}{p}, \frac{1}{p}}\right)} \\
& \quad+\left\|R \cdot \nabla u_{L}\right\|_{L_{t}^{1}\left(B_{p}^{-1+\frac{2}{p}, \frac{1}{p}}\right)}+\left\|u_{L} \cdot \nabla R\right\|_{L_{t}^{1}\left(B_{p}^{-1+\frac{2}{p}, \frac{1}{p}}\right)}+\|(1+a) \nabla P\|_{L_{t}^{1}\left(B_{p}^{-1+\frac{2}{p}, \frac{1}{p}}\right)} .
\end{aligned}
$$

It follows from the law of product, Lemma 2.2, that

$$
\|R \cdot \nabla R\|_{L_{t}^{1}\left(B_{p}^{-1+\frac{2}{p}, \frac{1}{p}}\right)} \lesssim\|R\|_{\tilde{L}_{t}^{\infty}\left(B_{p}^{-1+\frac{2}{p}, \frac{1}{p}}\right)}\left(\left\|\nabla_{\mathrm{h}} R\right\|_{L_{t}^{1}\left(B_{p}^{\frac{2}{p}, \frac{1}{p}}\right)}+\left\|\partial_{3} R\right\|_{L_{t}^{1}\left(B_{p}^{\frac{2}{p}}, \frac{1}{p}\right)}\right) .
$$

Yet we observe from interpolation inequality that

$$
\|R\|_{L_{t}^{1}\left(B_{p}^{\left.\frac{2}{p}, 1+\frac{1}{p}\right)}\right.} \lesssim \int_{0}^{t}\left\|R\left(t^{\prime}\right)\right\|_{B_{p}^{1+\frac{2}{p}, \frac{1}{p}}}^{\frac{1}{2}}\left\|R\left(t^{\prime}\right)\right\|_{B_{p}^{-1+\frac{2}{p}, 2+\frac{1}{p}}}^{\frac{1}{2}} d t^{\prime} .
$$


As a result, it comes out

$$
\|R \cdot \nabla R\|_{L_{t}^{1}\left(B_{p}^{-1+\frac{2}{p}, \frac{1}{p}}\right)} \lesssim\|R\|_{\tilde{L}_{t}^{\infty}\left(B_{p}^{-1+\frac{2}{p}, \frac{1}{p}}\right)}\left(\|R\|_{L_{t}^{1}\left(B_{p}^{1+\frac{2}{p}, \frac{1}{p}}\right)}+\|R\|_{L_{t}^{1}\left(B_{p}^{-1+\frac{2}{p}, 2+\frac{1}{p}}\right)}\right) .
$$

Along the same line, we have

$$
\begin{aligned}
\left\|u_{L} \cdot \nabla R\right\|_{B_{p}^{-1+\frac{2}{p}, \frac{1}{p}}} & \lesssim\left\|u_{L}\right\|_{B_{p}^{\frac{2}{p}, \frac{1}{p}}}\|\nabla R\|_{B_{p}^{-1+\frac{2}{p}, \frac{1}{p}}} \\
& \left.\lesssim\left\|u_{L}\right\|_{B_{p}^{-1+\frac{2}{p}, \frac{1}{p}}}^{\frac{1}{2}}\left\|\nabla_{\mathrm{h}} u_{L}\right\|_{B_{p}^{\frac{2}{p}, \frac{1}{p}}}^{\frac{1}{2}}\left\|\nabla_{\mathrm{h}} R\right\|_{B_{p}^{-1+\frac{2}{p}, \frac{1}{p}}}+\left\|\partial_{3} R\right\|_{B_{p}^{-1+\frac{2}{p}, \frac{1}{p}}}\right) \\
& \lesssim\left\|u_{L}\right\|_{B_{p}^{-1+\frac{2}{p}, \frac{1}{p}}}\left\|\nabla_{\mathrm{h}} u_{L}\right\|_{B_{p}^{\frac{2}{p}, \frac{1}{p}}}+\left\|\nabla_{\mathrm{h}} R\right\|_{B_{p}^{-1+\frac{2}{p}, \frac{1}{p}}}^{2}+\left\|\partial_{3} R\right\|_{B_{p}^{-1+\frac{2}{p}, \frac{1}{p}}}^{2}
\end{aligned}
$$

Notice that

$$
\begin{aligned}
& \left\|\nabla_{\mathrm{h}} R\right\|_{B_{p}^{-1+\frac{2}{p}, \frac{1}{p}}}^{2} \lesssim\|R\|_{B_{p}^{\frac{2}{p}, \frac{1}{p}}}^{2} \lesssim\|R\|_{B_{p}^{-1+\frac{2}{p}, \frac{1}{p}}}\|R\|_{B_{p}^{1+\frac{2}{p}, \frac{1}{p}{ }^{\prime}}} \\
& \left\|\partial_{3} R\right\|_{B_{p}^{-1+\frac{2}{p}, \frac{1}{p}}}^{2} \lesssim\|R\|_{B^{-1+\frac{2}{p}, 1+\frac{1}{p}}}^{2} \lesssim\|R\|_{B_{p}^{-1+\frac{2}{p}, \frac{1}{p}}}\|R\|_{B_{p}^{-1+\frac{2}{p}, 2+\frac{1}{p}}},
\end{aligned}
$$

so that there holds

$$
\begin{aligned}
\left\|u_{L} \cdot \nabla R\right\|_{L_{t}^{1}\left(B_{p}^{\left.-1+\frac{2}{p}, \frac{1}{p}\right)}\right.} \lesssim & \left\|u_{L}\right\|_{\tilde{L}_{t}^{\infty}\left(B_{p}^{-1+\frac{2}{p}, \frac{1}{p}}\right)}\left\|\nabla_{\mathrm{h}} u_{L}\right\|_{L_{t}^{1}\left(B_{p}^{\frac{2}{p}, \frac{1}{p}}\right)} \\
& +\|R\|_{\tilde{L}_{t}^{\infty}\left(B_{p}^{\left.-1+\frac{2}{p}, \frac{1}{p}\right)}\right.}\left(\|R\|_{L_{t}^{1}\left(B_{p}^{1+\frac{2}{p}, \frac{1}{p}}\right)}+\|R\|_{L_{t}^{1}\left(B_{p}^{-1+\frac{2}{p}, 2+\frac{1}{p}}\right)}\right) .
\end{aligned}
$$

Obviously, we have

$$
\left\|R \cdot \nabla u_{L}\right\|_{L_{t}^{1}\left(B_{p}^{\left.-1+\frac{2}{p}, \frac{1}{p}\right)}\right.} \lesssim \int_{0}^{t}\left\|R\left(t^{\prime}\right)\right\|_{B_{p}^{-1+\frac{2}{p}, \frac{1}{p}}}\left(\left\|\nabla_{\mathrm{h}} u_{L}\left(t^{\prime}\right)\right\|_{B_{p}^{\frac{2}{p}, \frac{1}{p}}}+\left\|\partial_{3} u_{L}\left(t^{\prime}\right)\right\|_{B_{p}^{\frac{2}{p}, \frac{1}{p}}}\right) d t^{\prime} .
$$

By substituting the above estimates and (4.1) into (4.3), and using [4, Lemma 4.3], we achieve

$$
\begin{aligned}
& \|R\|_{\tilde{L}_{t}^{\infty}\left(B_{p}^{-1+\frac{2}{p}, \frac{1}{p}}\right)}+v_{\mathrm{h}}\|R\|_{L_{t}^{1}\left(B_{p}^{1+\frac{2}{p}, \frac{1}{p}}\right)}+v_{\mathrm{V}}\|R\|_{L_{t}^{1}\left(B_{p}^{-1+\frac{2}{p}, 2+\frac{1}{p}}\right)} \\
& \lesssim\|R\|_{\tilde{L}_{t}^{\infty}\left(B_{p}^{-1+\frac{2}{p}, \frac{1}{p}}\right)}\left(\|R\|_{L_{t}^{1}\left(B_{p}^{1+\frac{2}{p}, \frac{1}{p}}\right)}+\|R\|_{L_{t}^{1}\left(B_{p}^{\left.-1+\frac{2}{p}, 2+\frac{1}{p}\right)}\right)}\right. \\
& +\|a\|_{\tilde{L}_{t}^{\infty}\left(B_{p}^{\frac{3}{p}}\right)}\left(v_{\mathrm{h}}\|R\|_{L_{t}^{1}\left(B_{p}^{1+\frac{2}{p}, \frac{1}{p}}\right)}+v_{\mathrm{V}}\|R\|_{L_{t}^{1}\left(B_{p}^{-1+\frac{2}{p}, 2+\frac{1}{p}}\right)}\right)
\end{aligned}
$$




$$
\begin{aligned}
& +\|a\|_{\tilde{L}_{t}^{\infty}\left(B_{p}^{\frac{3}{p}}\right)}\left(v_{\mathrm{h}}\left\|\Delta_{\mathrm{h}} u_{L}\right\|_{L_{t}^{1}\left(B_{p}^{-1+\frac{2}{p}, \frac{1}{p}}\right)}+v_{\mathrm{v}}\left\|\partial_{3}^{2} u_{L}\right\|_{L_{t}^{1}\left(B_{p}^{-1+\frac{2}{p}, \frac{1}{p}}\right)}\right) \\
& +\left\|u_{L}\right\|_{\tilde{L}_{t}^{\infty}\left(B_{p}^{-1+\frac{2}{p}, \frac{1}{p}}\right)}\left(\left\|\nabla_{\mathrm{h}} u_{L}\right\|_{L_{t}^{1}\left(B_{p}^{\frac{2}{p}, \frac{1}{p}}\right)}+\left\|\partial_{3} u_{L}\right\|_{L_{t}^{1}\left(B_{p}^{\frac{2}{p}, \frac{1}{p}}\right)}\right) \\
& +\int_{0}^{t}\left\|R\left(t^{\prime}\right)\right\|_{B_{p}^{-1+\frac{2}{p}, \frac{1}{p}}}\left(\left\|\nabla_{\mathrm{h}} u_{L}\left(t^{\prime}\right)\right\|_{B_{p}^{\frac{2}{p}, \frac{1}{p}}}+\left\|\partial_{3} u_{L}\left(t^{\prime}\right)\right\|_{B_{p}^{\frac{2}{p}, \frac{1}{p}}}\right) d t^{\prime} .
\end{aligned}
$$

Let us denote

$$
\begin{aligned}
T^{\star} \stackrel{\text { def }}{=} \sup \{ & t<T^{*}: v_{\mathrm{h}}\|a\|_{\tilde{L}_{t}^{\infty}\left(B_{p}^{\frac{3}{p}}\right)}+\|R\|_{\tilde{L}_{t}^{\infty}\left(B_{p}^{-1+\frac{2}{p}, \frac{1}{p}}\right)} \\
& \left.+v_{\mathrm{h}}\|R\|_{L_{t}^{1}\left(B_{p}^{1+\frac{2}{p}, \frac{1}{p}}\right)}+v_{\mathrm{v}}\|R\|_{L_{t}^{1}\left(B_{p}^{-1+\frac{2}{p}, 2+\frac{1}{p}}\right)} \leq 2 c_{0} v_{\mathrm{h}}\right\},
\end{aligned}
$$

where $c_{0}$ is a small enough positive constant, which will be fixed later on. Then for $t \leq T^{\star}$, one has

$$
\begin{aligned}
& \|R\|_{\tilde{L}_{t}^{\infty}\left(B_{p}^{-1+\frac{2}{p}, \frac{1}{p}}\right)}\left(\|R\|_{L_{t}^{1}\left(B_{p}^{1+\frac{2}{p}, \frac{1}{p}}\right)}+\|R\|_{L_{t}^{1}\left(B_{p}^{-1+\frac{2}{p}, 2+\frac{1}{p}}\right)}\right) \leq 2 c_{0}\|R\|_{\tilde{L}_{t}^{\infty}\left(B_{p}^{-1+\frac{2}{p}, \frac{1}{p}}\right)} \\
& \|a\|_{\tilde{L}_{t}^{\infty}\left(B_{p}^{\frac{3}{p}}\right)}\left(v_{\mathrm{h}}\|R\|_{L_{t}^{1}\left(B_{p}^{1+\frac{2}{p}, \frac{1}{p}}\right)}+v_{\mathrm{v}}\|R\|_{L_{t}^{1}\left(B_{p}^{-1+\frac{2}{p}, 2+\frac{1}{p}}\right)}\right) \leq 2 c_{0} v_{\mathrm{h}}\|a\|_{\tilde{L}_{t}^{\infty}\left(B_{p}^{\frac{3}{p}}\right)} .
\end{aligned}
$$

By substituting (4.7) and (4.8) into (4.5), and using Lemma 2.3, we achieve

$$
\begin{aligned}
& \|R\|_{\tilde{L}_{t}^{\infty}\left(B_{p}^{-1+\frac{2}{p}, \frac{1}{p}}\right)}+v_{\mathrm{h}}\|R\|_{L_{t}^{1}\left(B_{p}^{1+\frac{2}{p}, \frac{1}{p}}\right)}+v_{\mathrm{v}}\|R\|_{L_{t}^{1}\left(B_{p}^{-1+\frac{2}{p}, 2+\frac{1}{p}}\right)} \\
& \leq C\left(v_{\mathrm{h}}\|a\|_{\tilde{L}_{t}^{\infty}\left(B_{p}^{\frac{3}{p}}\right)}+\frac{v_{\mathrm{h}}^{1-\theta}}{v_{\mathrm{v}}^{1-\theta}}\|a\|_{\tilde{L}_{t}^{\infty}\left(B_{p}^{\frac{3}{p}}\right)}\left\|u_{0}\right\|_{B_{2}^{\frac{1}{2}}}\right. \\
& \quad+\|a\|_{\tilde{L}_{t}^{\infty}\left(B_{p}^{\frac{3}{p}}\right)}\left\|u_{0}\right\|_{B_{2}^{0, \frac{1}{2}}}+\frac{1}{v_{\mathrm{h}}^{\theta} v_{\mathrm{v}}^{1-\theta}}\left\|u_{0}\right\|_{B_{2}^{0, \frac{1}{2}}}\left(\left\|u_{0}\right\|_{B_{2}^{0, \frac{1}{2}}}+\left\|u_{0}\right\|_{B_{2}^{\frac{1}{2}}}\right) \\
& \left.\quad+\int_{0}^{t}\left\|R\left(t^{\prime}\right)\right\|_{B_{p}^{-1+\frac{2}{p}, \frac{1}{p}}}\left(\left\|\nabla_{\mathrm{h}} u_{L}\left(t^{\prime}\right)\right\|_{B_{p}^{\frac{2}{p}, \frac{1}{p}}}+\left\|\partial_{3} u_{L}\left(t^{\prime}\right)\right\|_{B_{p}^{\frac{2}{p}, \frac{1}{p}}}\right) d t^{\prime}\right) .
\end{aligned}
$$

Applying Gronwall inequality to (4.9) yields

$$
\begin{gathered}
\quad\|R\|_{\tilde{L}_{t}^{\infty}\left(B_{p}^{-1+\frac{2}{p}, \frac{1}{p}}\right)}+v_{\mathrm{h}}\|R\|_{L_{t}^{1}\left(B_{p}^{1+\frac{2}{p}, \frac{1}{p}}\right)}+v_{\mathrm{v}}\|R\|_{L_{t}^{1}\left(B_{p}^{-1+\frac{2}{p}, 2+\frac{1}{p}}\right)} \\
\leq\left(v_{\mathrm{h}}\|a\|_{\tilde{L}_{t}^{\infty}\left(B_{p}^{\frac{3}{p}}\right)}+\frac{v_{\mathrm{h}}^{1-\theta}\|a\|_{\tilde{L}_{t}^{\infty}\left(B_{p}^{\frac{3}{p}}\right)}\left\|u_{0}\right\|_{B_{2}^{\frac{1}{2}}}+\|a\|_{\tilde{L}_{t}^{\infty}\left(B_{p}^{\frac{3}{p}}\right)}\left\|u_{0}\right\|_{B_{2}^{0, \frac{1}{2}}}}{v_{\mathrm{v}}^{1-\theta} \|}\right.
\end{gathered}
$$




$$
\left.+\frac{1}{v_{\mathrm{h}}^{\theta} v_{\mathrm{v}}^{1-\theta}}\left\|u_{0}\right\|_{B_{2}^{0, \frac{1}{2}}}\left(\left\|u_{0}\right\|_{B_{2}^{0, \frac{1}{2}}}+\left\|u_{0}\right\|_{B_{2}^{\frac{1}{2}}}\right)\right) \exp \left(\frac{C\left(\left\|u_{0}\right\|_{B_{2}^{0, \frac{1}{2}}}+\left\|u_{0}\right\|_{B_{2}^{\frac{1}{2}}}\right)}{v_{\mathrm{h}}^{\theta} v_{\mathrm{v}}^{1-\theta}}\right) .
$$

On the other hand, by using interpolation inequality, we have

$$
\begin{aligned}
\|\nabla R\|_{L_{t}^{1}\left(L^{\infty}\right)} & \lesssim\|\nabla R\|_{L_{t}^{1}\left(B_{p}^{\frac{2}{p}, \frac{1}{p}}\right)} \lesssim\left\|\nabla_{\mathrm{h}} R\right\|_{L_{t}^{1}\left(B_{p}^{\frac{2}{p}, \frac{1}{p}}\right)}+\left\|\partial_{3} R\right\|_{L_{t}^{1}\left(B_{p}^{\frac{2}{p}, \frac{1}{p}}\right)} \\
& \lesssim\|R\|_{L_{t}^{1}\left(B_{p}^{1+\frac{2}{p}, \frac{1}{p}}\right)}+\|R\|_{L_{t}^{1}\left(B_{p}^{\frac{2}{p}, 1+\frac{1}{p}}\right)} \\
& \lesssim\|R\|_{L_{t}^{1}\left(B_{p}^{1+\frac{2}{p}, \frac{1}{p}}\right)}+\int_{0}^{t}\left\|R\left(t^{\prime}\right)\right\|_{B_{p}^{1+\frac{2}{p}, \frac{1}{p}}}\left\|R\left(t^{\prime}\right)\right\|_{B_{p}^{-1+\frac{2}{p}, 2+\frac{1}{p}}}^{\frac{1}{2}} d t^{\prime} .
\end{aligned}
$$

Then applying Young inequality, we get

$$
\|\nabla R\|_{L_{t}^{1}\left(L^{\infty}\right)} \lesssim\|R\|_{L_{t}^{1}\left(B_{p}^{1+\frac{2}{p}, \frac{1}{p}}\right)}+\|R\|_{L_{t}^{1}\left(B_{p}^{-1+\frac{2}{p}, 2+\frac{1}{p}}\right)} .
$$

So it follows from Lemma 2.4 that

$$
\begin{aligned}
\|a\|_{\tilde{L}_{t}^{\infty}\left(B_{p}^{\frac{3}{p}}\right)} \leq & \left\|a_{0}\right\|_{B_{p}^{\frac{3}{p}}} \exp \left(C^{\prime} \int_{0}^{t}\left\|\nabla u\left(t^{\prime}\right)\right\|_{L^{\infty}} d t^{\prime}\right) \\
\leq & \left\|a_{0}\right\|_{B_{p}^{\frac{3}{p}}} \exp \left(C ^ { \prime } \int _ { 0 } ^ { t } \left(\left\|\nabla_{\mathrm{h}} u_{L}\left(t^{\prime}\right)\right\|_{B_{p}^{\frac{2}{p}, \frac{1}{p}}}+\left\|\partial_{3} u_{L}\left(t^{\prime}\right)\right\|_{B_{p}^{\frac{2}{p}, \frac{1}{p}}}\right.\right. \\
& \left.\left.+\left\|\nabla_{\mathrm{h}} R\left(t^{\prime}\right)\right\|_{B_{p}^{\frac{2}{p}, \frac{1}{p}}}+\left\|\partial_{3} R\left(t^{\prime}\right)\right\|_{B_{p}^{\frac{2}{p}, \frac{1}{p}}}\right) d t^{\prime}\right) .
\end{aligned}
$$

Then for $t \leq T^{\star}$, we deduce from (2.4b), (2.4c), (4.11) and (4.6) that

$$
\|a\|_{\tilde{L}_{t}^{\infty}\left(B_{p}^{\frac{3}{p}}\right)} \leq\left\|a_{0}\right\|_{B_{p}^{\frac{3}{p}}} \exp \left(\frac{C\left(\left\|u_{0}\right\|_{B_{2}^{0, \frac{1}{2}}}+\left\|u_{0}\right\|_{B_{2}^{\frac{1}{2}}}\right)}{v_{\mathrm{h}}^{\theta} v_{\mathrm{v}}^{1-\theta}}\right) .
$$

Then we deduce from (4.10) that, under the small condition (1.5), for all $t \leq T^{\star}$ we obtain

$$
\begin{aligned}
& \|R\|_{\tilde{L}_{t}^{\infty}\left(B_{p}^{-1+\frac{2}{p}, \frac{1}{p}}\right)}+v_{\mathrm{h}}\|R\|_{L_{t}^{1}\left(B_{p}^{1+\frac{2}{p}, \frac{1}{p}}\right)}+v_{\mathrm{V}}\|R\|_{L_{t}^{1}\left(B_{p}^{-1+\frac{2}{p}, 2+\frac{1}{p}}\right)} \\
\leq & \left(v_{\mathrm{h}}\left\|a_{0}\right\|_{B_{p}^{\frac{3}{p}}}+\frac{v_{\mathrm{h}}^{1-\theta}}{v_{\mathrm{V}}^{1-\theta}}\left\|a_{0}\right\|_{B_{p}^{\frac{3}{p}}}\left\|u_{0}\right\|_{B_{2}^{\frac{1}{2}}}+\left\|a_{0}\right\|_{B_{p}^{\frac{3}{p}}}\left\|u_{0}\right\|_{B_{2}^{0, \frac{1}{2}}}\right.
\end{aligned}
$$




$$
\left.+\frac{1}{v_{\mathrm{h}}^{\theta} v_{\mathrm{v}}^{1-\theta}}\left\|u_{0}\right\|_{B_{2}^{0, \frac{1}{2}}}\left(\left\|u_{0}\right\|_{B_{2}^{0, \frac{1}{2}}}+\left\|u_{0}\right\|_{B_{2}^{\frac{1}{2}}}\right)\right) \exp \left(\frac{C\left(\left\|u_{0}\right\|_{B_{2}^{0, \frac{1}{2}}}+\left\|u_{0}\right\|_{B_{2}^{\frac{1}{2}}}\right)}{v_{\mathrm{h}}^{\theta} v_{\mathrm{v}}^{1-\theta}}\right) \leq c_{0} v_{\mathrm{h}}
$$

which contradicts the definition of $T^{\star}$. This in turn shows that $T^{\star}=T^{*}$. More precisely, thanks to (4.13), we infer

$$
\left\|\partial_{3} R\right\|_{L_{t}^{1}\left(B_{p}^{\frac{2}{p}, \frac{1}{p}}\right)} \lesssim\|R\|_{L_{t}^{1}\left(B_{p}^{1+\frac{2}{p}, \frac{1}{p}}\right)}^{\frac{1}{2}}\|R\|_{L_{t}^{1}\left(B_{p}^{\left.-1+\frac{2}{p}, 2+\frac{1}{p}\right)}\right.}^{\frac{1}{2}} \lesssim c_{0} v_{\mathrm{h}}^{\frac{1}{2}} \nu_{\mathrm{v}}^{-\frac{1}{2}},
$$

so that we deduce from (4.6) that

$$
\int_{0}^{T^{*}}\left(v_{\mathrm{h}}\left\|\nabla_{\mathrm{h}} R(t)\right\|_{L^{\infty}}+v_{\mathrm{v}}\left\|\partial_{3} R(t)\right\|_{L^{\infty}}\right) d t \leq C c_{0} v_{\mathrm{h}}^{\frac{1}{2}} \nu_{\mathrm{v}}^{\frac{1}{2}}
$$

And we also have

$$
\begin{aligned}
& \|R\|_{L_{t}^{2}\left(L^{\infty}\right)}^{2} \lesssim\|R\|_{L_{t}^{2}\left(B_{p}^{\left.\frac{2}{p}, \frac{1}{p}\right)}\right.}^{2} \lesssim \int_{0}^{t}\left\|R\left(t^{\prime}\right)\right\|_{B_{p}^{-1+\frac{2}{p}, \frac{1}{p}}}\left\|R\left(t^{\prime}\right)\right\|_{B_{p}^{1+\frac{2}{p}, \frac{1}{p}}} d t^{\prime} \\
& \lesssim\|R\|_{L_{t}^{\infty}\left(B_{p}^{-1+\frac{2}{p}, \frac{1}{p}}\right)}\|R\|_{L_{t}^{1}\left(B_{p}^{1+\frac{2}{p}, \frac{1}{p}}\right)} \\
& \lesssim\|R\|_{\tilde{L}_{t}^{\infty}\left(B_{p}^{\left.-1+\frac{2}{p}, \frac{1}{p}\right)}\right.}\|R\|_{L_{t}^{1}\left(B_{p}^{1+\frac{2}{p}, \frac{1}{p}}\right)} \leq C c_{0}^{2} v_{\mathrm{h}} .
\end{aligned}
$$

Similarly, by Lemma 2.3, we have

$$
\begin{aligned}
& \left\|u_{L}\right\|_{L_{t}^{2}\left(L^{\infty}\right)}^{2} \lesssim\left\|u_{L}\right\|_{\tilde{L}_{t}^{\infty}\left(B_{p}^{-1+\frac{2}{p}, \frac{1}{p}}\right)}\left\|u_{L}\right\|_{L_{t}^{1}\left(B_{p}^{1+\frac{2}{p}, \frac{1}{p}}\right)} \lesssim \frac{\left\|u_{0}\right\|_{B_{2}^{0, \frac{1}{2}}\left\|u_{0}\right\|_{B_{2}^{\frac{1}{2}}}}}{v_{\mathrm{h}}^{\theta} v_{\mathrm{v}}^{1-\theta}}, \\
& \left\|\nabla u_{L}\right\|_{L_{t}^{1}\left(B_{p}^{\left.\frac{2}{p}, \frac{1}{p}\right)}\right.} \lesssim\left\|\nabla_{\mathrm{h}} u_{L}\right\|_{L_{t}^{1}\left(B_{p}^{\frac{2}{p}, \frac{1}{p}}\right)}+\left\|\partial_{3} u_{L}\right\|_{L_{t}^{1}\left(B_{p}^{\left.\frac{2}{p}, \frac{1}{p}\right)}\right.} \lesssim \frac{\left\|u_{0}\right\|_{B_{2}^{0, \frac{1}{2}}}+\left\|u_{0}\right\|_{B_{2}^{\frac{1}{2}}}}{v_{\mathrm{h}}^{\theta} v_{\mathrm{v}}^{1-\theta}} .
\end{aligned}
$$

Consequently, there holds

$$
\int_{0}^{T^{*}}\left(\|\nabla u(t)\|_{L^{\infty}}+\|u(t)\|_{L^{\infty}}^{2}\right) d t<+\infty
$$

Then by Proposition 3.1, we get $T^{*}=+\infty$. This completes the proof of Theorem 1.1 . 


\section{Acknowledgments}

The author would like to thank Professor Ping Zhang for guidance and patience.

\section{References}

[1] H. Bahouri, J.-Y. Chemin and R. Danchin, Fourier Analysis and Nonlinear Partial Differential Equations, Grundlehren der Mathematischen Wissenschaften, 343, SpringerVerlag, 2011.

[2] J.-Y. Chemin, M. Paicu and P. Zhang, Global large solutions to 3-D inhomogeneous Navier-Stokes system with one slow variable, J. Differ. Equ. 256(1) (2014), 223-252.

[3] J.-Y. Chemin and P. Zhang, On the global wellposedness to the 3-D incompressible anisotropic Navier-Stokes equations, Commun. Math. Phys. 272 (2007), 529-566.

[4] J.-Y. Chemin and P. Zhang, On the critical one component regularity for 3-D NavierStokes system, Ann. Sci. Éc. Norm. Supér. 49(4) (2016), 131-167.

[5] J.-Y. Chemin and P. Zhang, Inhomogeneous incompressible viscous flows with slowly varying initial data, J. Inst. Math. Jussieu 17(5) (2018), 1121-1172.

[6] R. Danchin, Local theory in critical spaces for compressible viscous and heat-conductive gases, Commun. Partial. Differ. Equ. 26(7-8) (2001), 1183-1233.

[7] R. Danchin, Density-dependent incompressible viscous fluids in critical spaces, Proc. Roy. Soc. Edinburgh Sect. A 133 (2003), 1311-1334.

[8] P.-L. Lions, Mathematical Topics in Fluid Mechanics. Vol. 1. Incompressible Models, Oxford Lecture Series in Mathematics and Its Applications 3, Oxford Science Publications, The Clarendon Press, Oxford University Press, 1996.

[9] Y. Liu and P. Zhang, Global well-posedness of 3-D anisotropic Navier-Stokes system with large vertical viscous coefficient, J. Funct. Anal. 279 (2020), 108736.

[10] M. Paicu, Équation anisotrope de Navier-Stokes dans des espaces critiques, Rev. Mat. Iberoamericana 21 (2005), 179-235.

[11] M. Paicu and P. Zhang, Global strong solutions to 3-D Navier-Stokes system with strong dissipation in one direction, Sciences in China 62 (2019), 1175-1204.

[12] M. Paicu, P. Zhang and Z. Zhang, Global unique solvability of inhomogeneous NavierStokes equation with bounded density, Commun. Partial. Differ. Equ. 38 (2013), 12081234.

[13] P. Zhang, Global Fujita-Kato solution of 3-D inhomogeneous incompressible Navier-Stokes system, Adv. Math. 363 (2020), 107007. 